\title{
Benzodiazepine: indicazioni terapeutiche e utilizzo nel paziente nefropatico
}

\author{
Maria Josè Sequenza ${ }^{1}$, Dorian Soru ${ }^{2}$, Alessandro Carrus ${ }^{2}$, Maria Maddalena Sedda ${ }^{2}$, \\ Simeone Andrulli ${ }^{3}$, Vincenzo Barbera ${ }^{4}$, Domenico Ferrara ${ }^{5}$, Francesco Logias ${ }^{6}$
}

\author{
${ }^{1}$ Farmacia di Atzara, Atzara (NU) \\ ${ }^{2}$ Rete di Nefrologia e Dialisi, ASL di Nuoro, Nuoro \\ ${ }^{3}$ Divisione di Nefrologia e Dialisi, P.O. A. Manzoni, Lecco \\ ${ }^{4}$ U.O.C. di Nefrologia e Dialisi, P.O. Colleferro, Colleferro (RM) \\ ${ }^{5}$ U.O. di Nefrologia, Ospedale Cervello, Palermo \\ ${ }^{6}$ Direttore Rete di Nefrologia e Dialisi, ASL di Nuoro, Nuoro
}

\begin{abstract}
BENZODIAZEPINES: THERAPEUTICAL INDICATIONS AND USE IN PATIENTS WITH RENAL DISEASE
Abstract. Benzodiazepines, consisting of a benzene ring joined to a diazepine ring and a phenyl radical, are a class of drugs mainly used for the treatment of anxiety symptoms. They increase the transmission of gamma-aminobutyric acid (a kind of endogenous anxiolytic) and, therefore, they have an anxiolytic, sleep-inducing or sedative, myorelaxing, anticonvulsive, and anesthetic effect. They have minimal interactions with other drugs, but too often they are used as a long-term therapy instead of being used only when they are really necessary. The Territorial Pharmacist can play an important role in solving this problem. In patients with renal disease it is important to consider that the pharmacokinetics of these drugs, and therefore the absorption, degradation, and excretion of both their active principles and their metabolites are altered factors in case of kidney failure. Patients with renal disease make use of benzodiazepines more than the general population; however in these patients it is necessary to reduce the dose of about one third of the maximum dose allowed in patients with normal renal function. Additional research is also needed to investigate the main reasons of benzodiazepines use by patients with kidney disease.
\end{abstract}

Key words: Benzodiazepines, Benzodiazepine and renal disease, Treatment of anxiety

Conflict of interest: None.

Financial support: None.

Ricevuto: 29 Novembre 2013; Accettato: 3 Dicembre 2013

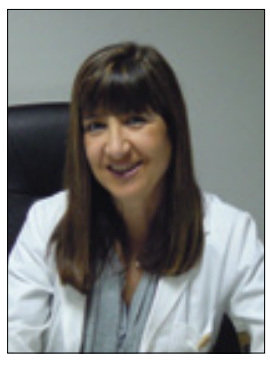

Maria Josè Sequenza

\section{Benzodiazepine: descrizione e principali indicazioni terapeutiche}

Le benzodiazepine sono costituite da un anello benzenico unito a un anello diazepinico e a un radicale fenilico. A tutt'oggi, esiste sul mercato italiano circa una trentina di benzodiazepine, classificate come ansiolitiche o ipnotiche, a seconda del dosaggio. Vengono identificate in base alla struttura chimica o all'emivita plasmatica. Nel primo caso, possono essere suddivise in 1,4 benzodiazepine, 1,5 benzodiazepine, triazolobenzodiazepine e imidazobenzodiazepine, mentre, nella suddivisione più comune basata sull'emivita plasmati- ca t1,2., si distinguono in benzodiazepine a lunga durata d'azione (emivita maggiore di 48 ore), benzodiazepine a durata d'azione intermedia (emivita compresa tra le 24 e le 48 ore), benzodiazepine a breve durata d'azione (emivita minore di 24 ore) e benzodiazepine a durata d'azione brevissima (emivita da 1 a 7 ore).

I diversi effetti terapeutici delle benzodiazepine sono dovuti alla capacità di incrementare la trasmissione acido gammaamminobutirrico / GABAergica: ansiolitico, ipnoinducente o sedativo, miorilassante, anticonvulsivante e anestetico generale (Fig. 1). Non vi sono molecole con un effetto, per esempio, solo miorilassante, ma potranno essere principalmente a effetto miorilassante e avere secondariamente tutti gli altri effetti, con gradi leggermente minori.

Le benzodiazepine agiscono amplificando le azioni del GABA, un neurotrasmettitore con un'influenza sedativa na- 


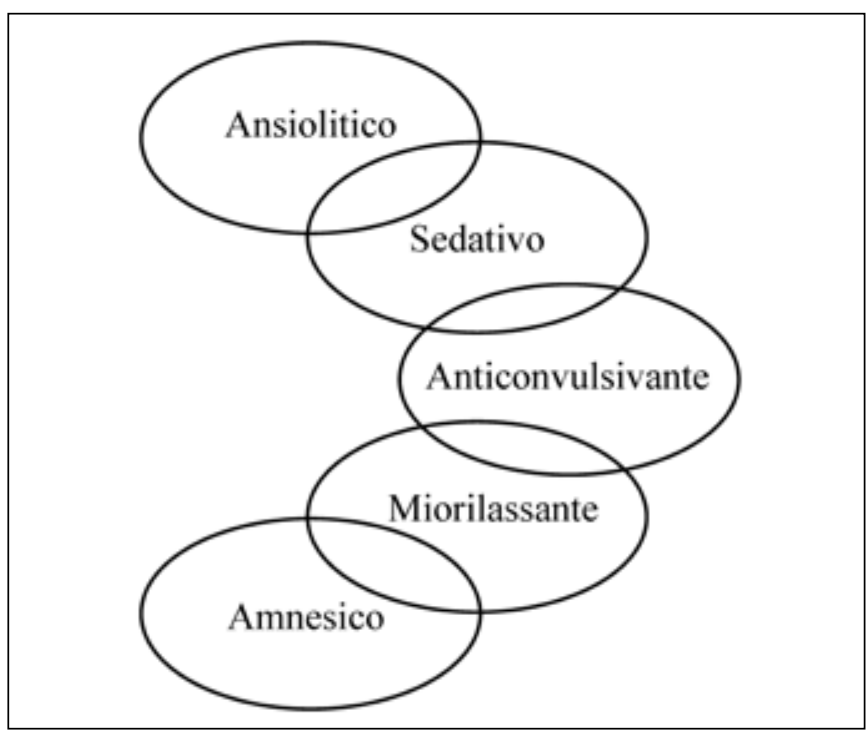

Fig. 1 - Effetti farmacologici delle benzodiazepine.

turale, generando, così, un'influenza inibitoria supplementare sui neuroni.

Le benzodiazepine sono utilizzate nel trattamento delle reazioni ansiose traumatiche acute o nell'ansia generalizzata con risoluzione in poche settimane, poiché riducono l'ansia e migliorano la qualità di vita del paziente, con minimi effetti collaterali. Vengono, altresì, utilizzate nel trattamento dell'insonnia occasionale o transitoria, nella prevenzione del delirium tremens e di altre manifestazioni di astinenza da alcolici, nel rilassamento muscolare dovuto agli effetti inibitori sui riflessi polisinaptici e sulla trasmissione internunciale, nel trattamento dell'epilessia con azione selettiva clinicamente utile nel trattamento degli stati convulsivi, nella preanestesia per via endovenosa, in premedicazione e nella sedazione in alcune manovre diagnostiche o terapeutiche.

Le vie di somministrazione più comuni sono le vie orale, rettale ed endovenosa: dotate di un'alta liposolubilità hanno una buona distribuzione su tutti i tessuti, attraversano la barriera placentare e sono escrete nel latte e nella saliva, per cui se ne sconsiglia la somministrazione nel primo trimestre di gravidanza, poiché sono potenzialmente responsabili di malformazioni fetali e di una moderata depressione respiratoria, se somministrate nell'ultimo trimestre o durante il travaglio. Da valutare anche la dipendenza fisica e il rischio nel neonato di sintomi da astinenza. Hanno un'emivita variabile da 2 ore ad alcuni giorni. Il loro metabolismo avviene, per la maggior parte, a livello epatico e l'escrezione avviene principalmente per via urinaria (per circa 1'80\%) e fecale (per circa il 10\%).

\section{Principali effetti collaterali}

Il più comune effetto collaterale è la sonnolenza, che si manifesta nel 10\% circa dei pazienti. A dosi più elevate, gli effetti tossici possono presentarsi con letargia, astenia grave o segni che possono ricordare quelli dell'intossicazione acuta da alcol, mentre il sovradosaggio si manifesta con un aumento dell'attività che raramente porta alla morte, se non associato ad altri deprimenti del SNC compreso l'alcol.

Un recente studio prospettico su pazienti anziani condotto nel corso di 15 anni evidenzia, inoltre, che l'uso di benzodiazepine comporta un aumentato rischio di demenza (1).

La cimetidina, il disulfiram, l'isoniazide e i contraccettivi orali possono ridurre il metabolismo delle benzodiazepine. Come già accennato, l'assunzione di bevande alcoliche ne potenzia gli effetti, come anche l'uso concomitante di deprimenti del SNC. Recenti studi dimostrano la capacità di alcuni dei fitoterapici come la valeriana e la passiflora, assunte sotto forma di tisane, di potenziare gli effetti delle benzodiazepine. La tolleranza, cioè l'aumento del dosaggio per ottenere lo stesso effetto diminuito nel tempo, si può distinguere in farmacocinetica, caratterizzata da alterazioni del metabolismo o della biodisponibilità, $\mathrm{o}$ in farmacodinamica, con una ridotta sensibilità del complesso recettore-effettore (2). Alcuni studi riportano lo sviluppo di una tolleranza entro le 24 ore dall'inizio del trattamento (3). Per ciò che riguarda l'assunzione cronica, la maggior parte dei pazienti riporta una tolleranza agli effetti ansiolitici e non riduce il dosaggio quando il problema risulta superato. Ovviamente, la tolleranza cronica risulta favorita da un tempo prolungato della terapia (4). L'interruzione drastica dell'assunzione prolungata delle benzodiazepine porta alla tendenza a sviluppare astinenza, provocando una serie di disturbi quali insonnia, problemi gastrointestinali e ansia, fino ad allucinazioni e a convulsioni (5).

Il grado e i tempi della sindrome da astinenza variano secondo la benzodiazepina assunta: le benzodiazepine a breve durata d'azione, come il triazolam, assorbite rapidamente e prive di metaboliti attivi grazie al loro metabolismo, possono presentare effetti di astinenza entro poche ore dall'ultima somministrazione, mentre le benzodiazepine a lunga durata d'azione, come il diazepam, possono presentarli in 2-6 giorni, con l'aggravamento dei sintomi alla seconda settimana.

Le molecole più utilizzate sono lorazepam, alprazolam, bromazepam e diazepam. È prassi comune utilizzare, per i casi di ansia, quando è necessaria una terapia farmacologica, le benzodiazepine a rapida eliminazione (lorazepam, oxazepam, alprazolam), per evitare un accumulo e avere minori effetti collaterali e un minore impegno metabolico da parte degli organi emuntori. Per ciò che riguarda, invece, il trattamento dell'insonnia, sono più indicate le benzodiazepine con rapidità sia di azione che di eliminazione, soprattutto per i soggetti che non hanno bisogno di una sedazione diurna e che devono mantenere un alto stato di veglia. Per i soggetti che presentano problemi sia di ansia che di insonnia può essere utilizzata la stessa benzodiazepina aumentando le dosi per la notte.

Da un'indagine sulla popolazione generale (255 pazienti) che si è recata in farmacia per acquistare una benzodiazepina per una terapia a lungo termine, effettuata nel distretto di Sorgono, nella Sardegna centrale, è emerso che il $65.88 \%$ dei pazienti intervistati è di sesso femminile, che l'età media è di 63 anni, che la prima prescrizione è stata fatta dal medico specialista nella misura del $64.62 \%$ rispetto a quella del medico di medicina generale, che continua la prescrizione con cadenza mensile. La benzodiazepina viene utilizzata come sedativo nel $59.70 \%$ dei casi e il tempo medio di terapia benzodiazepini- 
ca supera, in molti casi, i 10 anni. Le molecole più utilizzate sono l'alprazolam nella misura del $40.08 \%$, il lorazepam nella misura del $23.88 \%$, il bromazepam nella misura del $9.71 \%$ e il delorazepam nella misura del $7.69 \%$, seguiti dal prazepam e dal clonazepam.

Dagli studi, quindi, emerge come sia diffuso l'utilizzo delle benzodiazepine nelle terapie a lungo termine, pratica considerata non approvata dalle Linee Guida internazionali.

Il più grosso problema, però, degli effetti indesiderati delle benzodiazepine è, appunto, senza ombra di dubbio, l'inadeguatezza prescrittiva delle stesse. La prescrizione delle benzodiazepine come ansiolitici in monoterapia è molto più comune da parte dei medici di medicina generale che degli specialisti. È necessario ricordare che le benzodiazepine sono farmaci classificati nella classe $\mathrm{C}$, cioè a totale carico del paziente, e che sono dispensabili con ricetta ripetibile valida solamente 30 giorni per un massimo di tre pezzi. Le benzodiazepine sono classificate al primo posto come ansiolitici ipnotico-inducenti, sia come spesa che come numero di confezioni. Negli ultimi anni, l'uso delle benzodiazepine, nella maggior parte dei casi, è un vero e proprio abuso, complice anche la disponibilità prescrittiva di alcuni medici. Il loro costo contenuto, pochissimi euro, e "l'accondiscendenza" di alcuni farmacisti che non pretendono la ricetta medica contribuiscono alla posizione in "classifica".

Secondo uno studio condotto dall'INCB (International Narcotics Control Board), l'Italia, con una percentuale del 32\% della produzione globale, è anche tra i principali Paesi produttori di benzodiazepine. Secondo il United Nations Office for Drug Control and Crime Prevention, il 96\% dei Paesi che segnalano problemi di abuso mette al quarto posto le benzodiazepine $(69 \%)$ precedute da cocaina, oppioidi e cannabis (6).

Si denota, in generale, una crescita preoccupante della spesa totale per i farmaci a pagamento, che, oltretutto, contengono un numero abbastanza elevato di unità posologiche e hanno un costo molto contenuto.

La CTS (Commissione Tecnico-Scientifica) ha ritenuto necessario, anche per queste ragioni, procedere all'adeguamento degli stampati di specialità medicinali contenenti benzodiazepine. Nelle indicazioni terapeutiche, infatti, "le benzodiazepine sono indicate come ansiolitici soltanto quando il disturbo è grave e disabilitante o sottopone il soggetto a un grave disagio", casi in cui le indicazioni per la posologia indicano che il trattamento dovrebbe essere il più breve possibile. Il paziente dovrebbe essere monitorato regolarmente e la necessità di un trattamento continuato dovrebbe essere valutata attentamente, particolarmente se il paziente è senza sintomi. La durata complessiva del trattamento, generalmente, non dovrebbe superare le 8-12 settimane, compreso un periodo di sospensione graduale. Nel caso, invece, di insonnia il trattamento dovrebbe essere il più breve possibile: "la durata del trattamento varia da pochi giorni a due settimane, fino a un massimo di quattro settimane, compreso un periodo di sospensione graduale".

\section{Sospensione del trattamento con benzodiazepine}

La sospensione del trattamento con benzodiazepine è molto difficile e avviene molto raramente in caso di stati d'ansia o di problemi psichiatrici. Nell'insonnia è più semplice sospenderne l'uso, sfruttando alcuni accorgimenti che vedremo a breve. Può capitare, però, che, in occasioni particolari come interventi chirurgici, lutti o altre gravi situazioni, la sospensione brusca e autonoma possa causare nei soggetti che hanno sviluppato una dipendenza l'insorgenza di ansia, irritabilità, disforia, insonnia, tremori, tachicardia, cefalea e così via.

Per ovviare a questi inconvenienti, l'American Psychiatric Association (APA) suggerisce uno schema di sospensione concordato con il paziente. I punti fondamentali dello schema sono due; il primo riguarda la riduzione progressiva della dose:

- riduzione del 50\% della dose giornaliera con una certa rapidità;

- ulteriore riduzione del $25 \%$ della dose con una scansione temporale più ampia;

- $\quad$ eliminazione del rimanente $25 \%$ in un tempo molto lento. Bisogna, inoltre, fare attenzione alla riduzione delle dosi e ad allungare i tempi di intervallo tra una variazione e l'altra del dosaggio, quando si ha a che fare con benzodiazepine a breve emivita. Questo protocollo di sospensione può realizzarsi, in casi non particolarmente difficili, in 1-2 mesi.

Il secondo punto suggerito dall'APA è quello di sostituire una benzodiazepina a più lunga emivita rispetto a quella già utilizzata.

Il cambiamento di abitudini e la correzione degli stili di vita sono, a volte, sufficienti per risolvere alcuni disturbi funzionali che necessitano di una terapia. Tali cambiamenti di abitudini permettono comunque di vivere meglio, favorendo un corretto equilibrio psicofisico. Tra gli accorgimenti si segnalano in particolare:

- attività fisica moderata, meglio se all'aria aperta;

- una corretta alimentazione, evitando i carboidrati complessi di sera i quali, inducendo una maggiore produzione di insulina, causano un'ipoglicemia notturna e l'interruzione del sonno per la necessità di altro cibo;

- consumo di alcol e caffè con moderazione;

- abolizione del fumo;

- $\quad$ pause per svolgere attività soddisfacenti e rilassanti.

Per una buona fetta, le benzodiazepine vengono utilizzate per la cura dell'insonnia e, nonostante se ne raccomandi l'uso per brevi periodi, i tempi di terapia sono molto dilatati. In questi casi, si suggerisce la soluzione del problema con un trattamento non farmacologico, ove possibile, seguendo alcuni accorgimenti che possono migliorare la quantità e la qualità del sonno:

- $\quad$ andare a letto esclusivamente quando si sente di avere sonno;

- non trattenersi a letto con la luce accesa leggendo o guardando la televisione;

- $\quad$ se, entro un determinato lasso di tempo, il sonno non sopraggiunge alzarsi dal letto e rilassarsi;

- evitare i sonnellini durante la giornata;

- la sera, prima di coricarsi, concedersi un momento di relax; 
- $\quad$ smettere di lavorare o di svolgere attività interessanti o impegnative almeno 90 minuti prima di andare a dormire; - $\quad$ se non si riesce a prendere sonno, prima di andare a letto, sistemarsi in un luogo tranquillo e annotare le possibili soluzioni ai problemi insoluti.

Questo approccio terapeutico non farmacologico rappresenta una vera e propria strategia nella soluzione dell'insonnia, la cui efficacia è dimostrata in diversi lavori (7).

$\mathrm{Nel}$ caso in cui si verifichino casi di insonnia isolata è consigliabile ricorrere a un farmaco a breve emivita, con rapida distribuzione e rapida eliminazione, come il temazepam, che induce velocemente il sonno, viene metabolizzato entro la mattina successiva ed evita risvegli nel cuore della notte, come succederebbe con l'assunzione di benzodiazepine a emivita ultrabreve.

La psicoterapia e/o i rimedi naturali, tra i più utilizzati e conosciuti la valeriana, la passiflora, il biancospino e l'iperico, possono, a volte, risolvere il problema con una minore spesa e minori effetti collaterali, soprattutto in particolari pazienti, come i nefropatici, con un sistema emuntore già compromesso.

\section{Benzodiazepine e patologia renale}

È molto importante, nell'utilizzo delle benzodiazepine nel paziente con una patologia renale, tenere in alta considerazione i prodotti di degradazione e l'escrezione sia dei principi attivi che dei loro metaboliti, fattori alterati nell'insufficienza renale. È bene ricordare, inoltre, che, per alcune benzodiazepine, esiste un ridotto assorbimento a livello del piccolo intestino, causato dall'eccessiva alcalinizzazione gastrica a volte associata alla patologia renale. Alcune situazioni concomitanti portano alla richiesta di un aumento o di una diminuzione della dose dei farmaci, come nel caso di ascite o disidratazione. Un altro fattore che può modificare le dosi richieste è, per esempio, il sesso: le donne, infatti, avendo una minore capacità di svuotamento gastrico e un tempo di transito intestinale più rapido (8), possono ridurre la biodisponibilità dei farmaci. Inoltre, nelle donne, le quali presentano un livello di acidità gastrica minore rispetto all'uomo, l'assunzione di farmaci a pH tendenzialmente basico come le benzodiazepine comporta che esse possano essere assorbite più velocemente e raggiungere concentrazioni più elevate in tempi minori (9); e, ancora, nelle persone obese soprattutto di sesso femminile, nonché negli anziani che, con il progredire dell'età, aumentano la massa grassa, con l'assunzione di farmaci che hanno un'alta affinità per i grassi come le benzodiazepine si verificano accumuli importanti con un aumento dell'emivita del farmaco $(10,11)$. Non esistono, comunque, degli schemi posologici standardizzati, poiché sia le dosi che i tempi di somministrazione devono essere personalizzati tenendo in considerazione le eventuali necessità del paziente. Quindi, sarebbe meglio attenersi a dosaggi non superiori ai due terzi della dose consentita per il paziente con funzionalità renale normale (12).

Alcuni studi hanno rilevato che una percentuale abbastanza elevata, tra il $38 \%$ e il $45 \%$, di pazienti con insufficienza renale terminale presenta problemi di ansia (13).

Nei pazienti che presentano un'alterazione della funzionalità renale, si evidenzia che l'assorbimento del delorazepam avviene in maniera relativamente più lenta rispetto agli altri pazienti, con una buona biodisponibilità (pari all'82\%). Nel caso dei pazienti geriatrici, dei bambini e dei pazienti con alterazioni della funzione epatica o renale, sarebbe opportuno prescrivere un dosaggio iniziale più basso, in modo da evitare i sintomi di una sedazione eccessiva che si potrebbe manifestare a causa del metabolismo rallentato in questo tipo di pazienti. Sono, inoltre, consigliabili verifiche frequenti dei valori ematici e della funzione renale, personalizzando le dosi. In un recente studio condotto in Veneto (14), si evidenzia che, su 3300 pazienti che hanno ricevuto almeno una prescrizione di benzodiazepine nei due anni della durata dello studio, il $24.3 \%$ si è rivolto al medico per un trattamento a lungo termine (almeno sei mesi di terapia). È stato possibile verificare che il principio attivo maggiormente prescritto è stato il lorazepam, che è risultato nettamente prevalente $(36.4 \%)$, seguito dall'alprazolam (19.2\%), dal bromazepam (14.9\%) e dal diazepam $(12.2 \%)$, e che circa un quarto dei pazienti trattati è stato in terapia per un periodo superiore ai sei mesi.

\section{Il ruolo del farmacista territoriale}

Una delle figure sanitarie più accreditate, che dovrebbe essere utilizzata per sfruttare pienamente le proprie qualità formative e professionali, per garantire la correttezza e la sicurezza di tutto l'iter terapeutico, è il farmacista clinico territoriale. Una nuova figura professionale nata dall'esigenza di una maggiore richiesta di competenze da parte del cittadino. Si offre, quindi, la disponibilità al Servizio Sanitario Nazionale di una professionalità altamente formata e qualificata nello specifico settore del farmaco, con alti livelli di garanzia e diminuzione dei rischi clinici. Si veda, per esempio, l'introduzione, con la Legge del 18 Giugno 2009, n. 69, della farmacia "nell'assistenza domiciliare integrata in collaborazione con le altre figure professionali sanitarie nel territorio" (come il medico di medicina generale) "con l'obiettivo di garantire il corretto utilizzo dei medicinali prescritti e il relativo monitoraggio, al fine di favorire l'aderenza dei malati alle terapie mediche". Il ruolo della farmacia pubblica è fondamentale nella promozione della salute e nella prevenzione e nell'educazione sanitaria del cittadino, per fornirgli un'adeguata informazione sull'uso più consapevole e corretto del farmaco, in modo da sfruttare al massimo le potenzialità dell'effetto farmacologico e ridurne i rischi dovuti all'errata assunzione. Inoltre, bisogna responsabilizzare il cittadino, informandolo sul fatto che tutti i farmaci hanno un costo, anche se erogati gratuitamente dal SSN. Lo scorretto uso del farmaco sottrae risorse che potrebbero essere sfruttate per la ricerca o per migliorare altri servizi. Diversi studi evidenziano che, mediamente, solo il $30-50 \%$ di tutti i farmaci prescritti per malattie croniche viene assunto dai pazienti seguendo le indicazioni dei medici prescrittori (15), con pesanti conseguenze sulla salute dei singoli individui e senza alcun miglioramento dello stato di salute, anzi con un aumento dell'ospedalizzazione e dei relativi costi. Questi ultimi includono non solo quelli dei medicinali inutilizzati, ma anche quelli aggiuntivi, come pensioni di invalidità, assistenza domiciliare e coinvolgimento dei familiari. È inutile, quindi, investire ingenti somme di denaro nella ri- 
cerca di terapie innovative, se poi il loro potenziale beneficio non viene sfruttato, a causa dello scorretto uso del farmaco da parte dei pazienti non monitorati. L'aderenza alla terapia è un problema abbastanza complicato e non di facile soluzione e già conosciuto da Ippocrate (IV sec. a.C.), quando scrisse: "Sorvegliate anche le colpe dei pazienti, che spesso li fanno mentire circa l'assunzione delle cose prescritte". Ancora oggi, il problema dell'aderenza terapeutica è considerato una causa di fallimento della terapia. Le cause di questo fallimento sono molteplici e non sempre correlabili al paziente (cfr. il "Manifesto per l'aderenza alla terapia farmacologica sul territorio italiano" presentato in Senato il 30/01/2013 durante un Convegno promosso dall'Associazione Parlamentare per la Tutela e la Promozione del Diritto alla Prevenzione). In tal senso, i farmacisti dell'Unione Europea stanno operando per accrescere la loro professionalità e migliorare la pratica farmaceutica, al fine di soddisfare i crescenti bisogni dei pazienti e di affrontare al meglio le attuali sfide del settore sanitario: "I governi europei stanno cercando di ottimizzare l'utilizzo del farmaco, allo scopo di migliorare la farmacoterapia e di ridurre i costi complessivi della sanità" (16).

\section{Conclusioni}

Concludendo, non va dimenticato che l'ansia in sé non è una patologia, ma svolge importantissime funzioni adattive, attivando il comportamento e ottimizzando le risposte agli stimoli esterni. Le benzodiazepine oxazepam-simili (lorazepam, temazepam) sono da preferire per la loro breve emivita plasmatica e per l'assenza di metaboliti farmacologicamente attivi. Le dosi da utilizzare nell'insufficienza renale vanno circa dalla metà a tre quarti di quelle utilizzate dai pazienti sani e devono essere, comunque, sempre personalizzate.

Il farmaco adatto alla patologia e alla persona, ben dosato, nonché un buon rapporto medico-paziente che consenta una "concordance" tra i due e ottimizzi gli effetti della terapia spesso sono una garanzia di risultato.

L'importante è che il paziente non sia abbandonato a se stesso; talvolta la terapia va dosata più volte, cambiata, se necessario, e adattata alle esigenze e alle specificità di ogni singolo paziente. Queste considerazioni diventano ancora più rilevanti nella patologia renale, dato il vasto uso che viene fatto di questi farmaci, per ragioni che ancora meritano un adeguato approfondimento.

\section{Riassunto}

Le benzodiazepine, costituite da un anello benzenico unito a un anello diazepinico e a un radicale fenilico, sono una classe di farmaci elettivamente utilizzati per il trattamento dei sintomi ansiosi. Esse incrementano la trasmissione dell'acido gamma-amminobutirrico (una sorta di ansiolitico endogeno) e, perciò, presentano un effetto ansiolitico, ipnoinducente o sedativo, miorilassante, anticonvulsivante e anestetico generale. Presentano minime interazioni farmacologiche con altri farmaci ma troppo spesso vengono utilizzate come terapia a lungo termine e non quando è strettamente necessario. Il farmacista territoriale può svolgere un ruolo sempre più importante per risolvere questo problema. Nel paziente con una patologia renale, occorre tenere in alta considerazione la farmacocinetica di questi farmaci e, quindi, l'assorbimento, la degradazione e l'escrezione sia dei principi attivi che dei loro metaboliti, fattori alterati nell'insufficienza renale. I pazienti con patologia renale fanno un uso di benzodiazepine maggiore rispetto alla popolazione generale, tuttavia è necessaria una riduzione del dosaggio di circa un terzo della dose massima consentita in pazienti con una funzione renale normale. Sono, inoltre, necessarie delle ricerche che indaghino sulle principali ragioni dell'utilizzo delle benzodiazepine da parte dei pazienti nefropatici.

Parole chiave: Benzodiazepine, Benzodiazepine e patologia renale, Trattamento dell'ansia

Dichiarazione di conflitto di interessi: Gli Autori dichiarano di non avere conflitto di interessi.

Contributi economici agli Autori: Gli Autori dichiarano di non avere ricevuto sponsorizzazioni economiche per la preparazione dell'articolo.

\author{
Indirizzo degli Autori: \\ Dr. Francesco Logias \\ Via Mannironi \\ 08100 Nuoro (NU) \\ logias@tiscali.it
}

\section{Bibliografia}

1. Billioti de Gage S, Bégaud B, Bazin F, et al. Benzodiazepine use and risk of dementia: prospective population based study. BMJ 2012; 345: e6231.

2. Hollister LE. Dependence on benzodiazepines. In: Szara SL, Ludford JP, ed. NIDA Res. Monograph 33, Rockville, Maryland 1980: 70-82.
3. Rosemberg HC, Chiu TH. Time course for development of benzodiazepine tolerance and physical dependence. Neurosci Biobehav Rev 1985; 9 (1): 123-31.

4. Khan A, Hornblow AR, Walshe JW. Benzodiazepine dependence: a general practice survey. N Z Med J 1981; 93 (687): 19-21.

5. American Psychiatric Association. Diagnostic and Statistical Manual of Mental Disorders DSM-IV, Fourth Edi- 
tion (Text Revision). Washington DC, American Psychiatric Association, 2000.

6. Lo Iacono S, ed, 2010 "Dossier Usi e abusi dei farmaci ansiolitici”. Disponibile su http://www.mdc.it/documenti/usi abusi dei farmaci ansiolitici_2010.pdf (Accesso il 15 Novembre 2013).

7. Bootzin RR, Perlis ML. Nonpharmacologic treatments of insomnia. J Clin Psychiatry 1992; 53: 37-41.

8. Rao SS, Read NW, Brown C, et al. Studies on the mechanism of bowel disturbance in ulcerative colitis. Gastroenterology 1987; 93 (5): 934-40.

9. Pollock BG. Gender differences in psychotropic drug metabolism. Psychopharmacol Bull 1997; 33 (2): 235-41.

10. Greenblatt DJ, Sellers EM, Shader RI. Drug therapy: drug disposition in old age. N Engl J Med 1982; 306 (18): 1081-8.

11. Sweet RA, Pollock BG, Kirshner M, et al. Pharmacoki- netics of single- and multiple-dose bupropion in elderly patients with depression. J Clin Pharmacol 1995; 35 (9): 876-84.

12. Brater DC. Drug Use In Renal Disease. ADIS Health Science Press, Balgowlah, Australia 1983; 22-56.

13. Murtagh FE, Addington-Hall J, Higginson IJ. The prevalence of symptoms in end-stage renal disease: a systematic review. Adv Chronic Kidney Dis 2007; 14 (1): 82-99.

14. Quaglio A, Quaglio G. L'uso delle benzodiazepine nella medicina generale. Medicina Pratica 2010; 4: 164-7.

15. Sabaté E. Adherence to long-term therapies: evidence for action. World Health Organization 2003.

16. Pharmaceutical Group of the European Union (PGEU), 2012 "Il libro blu della farmacia europea". Disponibile su http://www.federfarma.it/Documenti/farmacia_mondo/LibroBlu.aspx (Accesso il 15 Novembre 2013). 